\title{
Joint Annual Conference of the DGOR and the NSOR 1987
}

The joint annual conference of the DGOR and the NSOR will take place at the congress center Koningshof in Veldhoven near Eindhoven

\section{September 23 to 25,1987}

The intention of the conference is to inform about the latest developments in Operations Research in theory and practice and to establish a forum for exchanging experiences as well as for stimulating new activities.

The scientific program is planned to have survey lectures and special topics papers mostly concerned with the following topics.

- Operations Research in industry and trade, Banking and insurance, Capital expenditures and finance, Marketing, Applications from industrial practice

- Production planning and inventory, Flexible manufacturing systems, Logistics and traffic
- Decision support and expert systems, Strategic planning

- Organization and decision theory

- Data analysis and forecasting, Operations Research in Personal Computing

- Operations Research in health care

- Stochastic decision processes, Control theory, Reliability and queueing theory, Simulation

- Mathematical programming, Combinatorial optimization

Experience reports are especially invited. Conference languages are German and English.

It is intended to publish a compendium of the conference results including selected papers in full length. The compendium will be published by Springer-Verlag Berlin, Heidelberg, New York.

Program Committee:

\section{Chairman:}

Prof. Dr. Helmut Schellhaas

Fachbereich Mathematik

Technische Hochschule Darmstadt

Schloßgartenstraße 7

D-6100 Darmstadt

Tel.: (06151) 16-2288/-2188

Members:

Prof. Dr. Paul van Beek

Landbouwuniversiteit Wageningen

Prof. Dr. Heinz Isermann

Universität Bielefeld
Prof. Dr. Reinhart Schmidt Christian-Albrechts-Universität Kiel

Drs. Mynt Zijlstra

Nederlandse Philips Bedrijven B. V. Eindhoven

Organization Committee:

Prof. Dr. Paul van Beek

Landbouwuniversiteit Wageningen

De Dreijen 8

NL-6703 BC Wageningen

Prof. Dr. C. Bernhard Tilanus

Technische Universiteit Eindhoven
P.O. Box 513

NL-5600 MB Eindhoven

Prof. Dr. Jaap Wessels

Technische Universiteit Eindhoven

P.O. Box 513

NL-5600 MB Eindhoven

Office:

Lärchenweg 9

D-6916 Wilhelmsfeld

Tel.: (06220) 1223

DEUTSCHE GESELLSCHAFT FÜR OPERATIONS RESEARCH (DGOR)

NEDERLANDSE STICHTING VOOR OPERATIONS RESEARCH (NSOR)

Prof. Dr. D. Ohse (Chairman)/Prof. Dr. P. van Beek (Chairman) 